Draft Version November 7, 2018

Preprint typeset using $\mathrm{LAT}_{\mathrm{E}} \mathrm{X}$ style emulateapj v. 5/2/11

\title{
SENSITIVITY OF STACKED IMAGING DETECTORS TO HARD X-RAY POLARIZATION
}

\author{
FABio Muleri and Riccardo CAMPANA \\ INAF/IAPS, Via del Fosso del Cavaliere 100, I-00133 Roma, Italy \\ Draft version November 7, 2018
}

\begin{abstract}
The development of multi-layer optics which allow to focus photons up to $100 \mathrm{keV}$ and more promises an enormous jump in sensitivity in the hard X-ray energy band. This technology is already planned to be exploited by future missions dedicated to spectroscopy and imaging at energies $>10 \mathrm{keV}$, e.g. Astro-H and NuSTAR. Nevertheless, our understanding of the hard X-ray sky would greatly benefit from carrying out contemporaneous polarimetric measurements, because the study of hard spectral tails and of polarized emission often are two complementary diagnostics of the same non-thermal and acceleration processes. At energies above a few tens of $\mathrm{keV}$, the preferred technique to detect polarization involves the determination of photon directions after a Compton scattering. Many authors have asserted that stacked detectors with imaging capabilities can be exploited for this purpose. If it is possible to discriminate those events which initially interact in the first detector by Compton scattering and are subsequently absorbed by the second layer, the direction of scattering is singled out from the hit pixels in the two detectors. In this paper we give the first detailed discussion of the sensitivity of such a generic design to the X-ray polarization. The efficiency and the modulation factor are calculated analytically from the geometry of the instruments and then compared with the performance as derived by means of Geant4 Monte Carlo simulations.
\end{abstract}

Subject headings: X-ray — polarimetry — Compton scattering

\section{INTRODUCTION}

After the first pioneering experiments in the '70s (Novick et al. 1972; Weisskopf et al. 1976, 1978), only very few polarimetric measurements have been carried out in high energy astronomy (Coburn \& Boggs 2003; Rutledge \& Fox 2004; Willis et al. 2005; Kalemci et al. 2007; Dean et al. 2008; Forot et al. 2008; Yonetoku et al. 2011a). The main reason which prevented polarimetry to become a common tool also in this energy band was that even state-of-art instruments were able to measure the polarization of only the brightest X-ray sources. In the soft $\mathrm{X}$-ray energy range, where grazing incidence optics were available, Bragg diffraction polarimeters allowed only for a modest quantum efficiency, whereas Thomson scattering polarimeters had a energy threshold mismatched with the energy band pass of the telescopes (Novick 1975). At higher energies, without the possibility to focus X-rays, Compton polarimeters required a large collecting area and consequently the high background limited the sensitivity. As a result, no dedicated polarimeters after the one on-board the OSO- 8 satellite were launched, with the exception of the small polarimeter Gamma-Ray Burst Polarimeter (GAP) recently launched on-board the Japanese satellite IKAROS to observe with a large field of view the prompt emission of Gamma Ray Bursts (Yonetoku et al. 2011b).

Today polarimetry is a field of growing interest in high energy astrophysics (Bellazzini et al. 2010). At low energy, gas detectors able to image the path of the photoelectron in low atomic number mixtures, e.g. Helium or Neon and dimethyl ether (DME), are a valuable alternative to Bragg diffraction and Thomson scattering polarimeters (Costa et al. 2001; Black et al. 2007; Bellazzini \& Spandre 2010). The mission Gravity and Ex- treme Magnetism SMEX (GEMS), a small explorer satellite planned to be launched in 2014, exploits these photoelectric polarimeters together with grazing incidence telescopes and promises to perform polarimetry of sources as faint as a few mCrab between 2 and $10 \mathrm{keV}$, with an enormous improvement in sensitivity with respect to OSO-8 polarimeters (Black et al. 2010; Jahoda 2010). On the other hand, the recent development of multi-layer optics (Christensen et al. 1992; Pareschi \& Cotroneo 2003) makes attractive to extend this energy range upward. The energy range of photoelectric polarimeters can be extended above $10 \mathrm{keV}$ by using higher atomic number mixtures, like Argon and DME possibly at high pressure (Muleri et al. 2006; Soffitta et al. 2010), but above a few tens of keV Compton polarimeters (McConnell 2010) become more appealing because of the prevailing probability of Compton scattering with respect to photoabsorption.

The design of a Compton polarimeter is of the greatest importance both to achieve the best sensitivity and to reduce the systematic effects. Indeed, there are a number of very different proposals in the literature (see e.g. Krawczynski et al. 2011). In this paper we want to discuss the performance of a particular design which, although not dedicated to polarimetry, can provide some polarimetric sensitivity contemporaneously with imaging and spectroscopy. As a matter of fact, many authors have asserted that such a design would endow next imaging/spectroscopy X-ray missions with the capability to detect also polarization from bright sources (Barret et al. 2003; Gouiffès et al. 2008; Ferrando et al. 2010). The geometry of such an instrument will be described in Section 2, while the performance will be investigated with a full analytical treatment and with Geant4 simulations in Section 3 and in Section 4, respectively. 


\section{DESCRIPTION OF THE STACKED IMAGER POLARIMETER}

A common instrument layout dedicated to broad-band imaging in the focal plane of a X-ray telescope exploits two stacked pixelated detection planes designed to absorb photons at different energies (e.g. the imager camera onboard NHXM, Catalano et al. (2010), or the Wide Field Imager on-board the International X-ray Observatory, Stefanescu et al. (2010)). The first detector is usually made of Silicon and it is used to detect photons at lower energy ( $\lesssim 1$ to $\sim 15 \mathrm{keV}$, depending on the thickness of the depletion layer), while harder photons, up to $\sim 100 \mathrm{keV}$, are absorbed in the second detector, which is based on CdTe or CZT crystals. Although the largest part of the high energy photons passes through the first detection plane without interacting, a tiny fraction is absorbed or scattered by Silicon. For what concerns this paper, only the latter events are those interesting for measuring polarization. If the energy deposit due to the scattering in the Silicon is above the detection threshold and, after the scattering, the photons are absorbed by the second detection plane, the direction of scattering can be singled out by joining the hit pixels in the two detectors (see Figure 1).

Therefore, any instrument with two stacked imaging detectors has, besides imaging capabilities, also an intrinsic sensitivity to polarization. To exploit it, one must distinguish among the events which release an energy deposit in both detectors, that we call "double events", those in which the photon underwent a scattering in the first detection plane and was absorbed in the second one. A first distinctive feature of such "good" events is that the signals from the two detection planes are basically contemporaneous and therefore the LEID and the HEID must be put into coincidence. The time window must be short enough to make negligible the probability of accidental coincidences due to, for example, background or pile-up and this may require a coincidence window of a few $\mu$ s or less, and in any case a shaping time $\approx \mu \mathrm{s}$. Another constraint for an event to be a good Compton double event is that the energy released in the first detector must be below a certain threshold corresponding to the maximum accepted scattering angle. The threshold is below a few keV for incident photons with energy less than $40 \mathrm{keV}$ and reasonable values of the scattering angle.

The implementation of these constraints poses a number of requirements on the two detectors. Some of them, e.g. the fast read-out, are quite severe for the Siliconbased detection plane. Nonetheless they seem to be in the range of the current technology based on Silicon Drift Detectors (Lechner et al. 2010). In the following we will not discuss in more detail the actual feasibility of the instrument taken as an example in this paper, because our primary aim is to assess the intrinsic sensitivity to polarization of the stacked imager design. Therefore, we will naively assume a "toy model" including only the fundamental components. The first pixelated detection plane, hereafter called Low Energy Imaging Detector (LEID), is made of Silicon, while the second stage is made of CdTe crystals and will be referred to as High Energy Imaging Detector (HEID). The characteristics assumed for the LEID and for the HEID are reported in Table 1 and they are in line with those actually required in modern mission proposals. We will also suppose that the time coincidence between the two detectors is fast enough that the occurrence of spurious coincidences is negligible, as the background. Since the instrument discussed is designed to be used in the focal plane of a telescope, we will focus our attention in the energy range $20-100 \mathrm{keV}$, which is the interval where multilayer optics will be applied in the next years.

Table 1

Characteristics of the stacked imager polarimeter assumed throughout this paper.

\begin{tabular}{ll}
\hline \multicolumn{2}{l}{ Low Energy Imaging } \\
\hline Material & Detector (LEID) \\
Thickness & $450 \mu \mathrm{m}$ \\
Area & $51.2 \times 51.2 \mathrm{~mm}^{2}$ \\
Pixels & $512 \times 512$ pixels, square pattern \\
Pixel size & $100 \mu \mathrm{m} \times 100 \mu \mathrm{m}$ \\
\hline High Energy Imaging Detector (HEID) \\
\hline Material & CdTe \\
Thickness & $2 \mathrm{~mm}$ \\
Area & $51.2 \times 51.2 \mathrm{~mm}^{2}$ \\
Pixels & $256 \times 256 \mathrm{pixels}$, square pattern \\
Pixel size & $200 \mu \mathrm{m} \times 200 \mu \mathrm{m}$ \\
\hline Distance LEID/HEID & $2 \mathrm{~cm}$ \\
Energy range & $20-100 \mathrm{keV}$ \\
\hline
\end{tabular}

In the following, it will be implicitly assumed that the Minimum Detectable Polarization, that is the upper limit to the polarization which can be statistically measured in a certain observation time and with a certain confidence, is fully representative of the sensitivity of the instrument. This approach is purely statistical and, consequently, it will neglect a very important requirement of any actual polarimeter, i.e. the reduction to a negligible level and, if this is not possible, the correction of any systematic effects which may result in a spurious polarized signal. Systematics may be severe for the polarimeter discussed here, because the probability of scattering in the first detection plane is tiny and therefore there are a number of small effects which may play a role. Without the intention to be complete, systematic effects may arise from nonuniformities in the pixel efficiency, energy threshold or geometry, or they may come out from pointing instabilities or anisotropic background. These issues must be extensively studied if dealing with the feasibility of the polarimeter design discussed in this paper, but, again, this is out of the scope of this work where we rather focus on the "bulk" of the problem, that is the performance of the method in ideal conditions.

\section{SENSITIVITY TO POLARIZATION - ANALYTICAL TREATMENT}

Compton polarimeters detect the polarization of the incident radiation by measuring the photon scattering direction: a polarized signal causes a square-cosine modulation to appear in the histogram of azimuthal directions of scattering (hereafter modulation curve). Even if the incident radiation is completely unpolarized, the number of photons scattered per angular bin is Poissondistributed around the mean value, and this always mimics a spurious modulation to some extent. The amount of this modulation at a certain confidence level translates in 


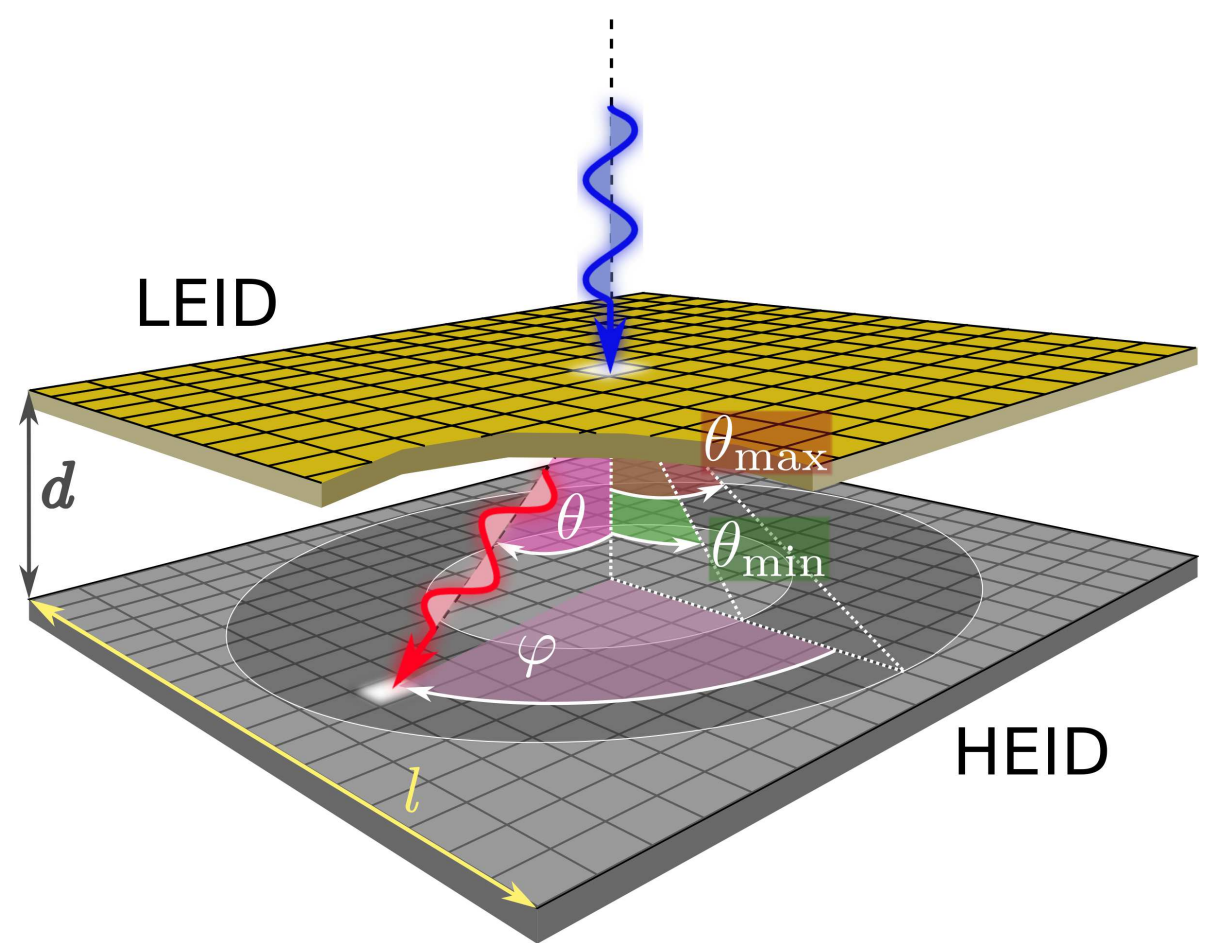

Figure 1. Layout of the polarimeter studied in this paper. Polarization of photons is measured by selecting those events which are scattered by the first detection plane (named LEID, Low Energy Imaging Detector) and subsequently absorbed by the High Energy Imaging Detector (HEID). The scattering angle $\theta$ and the azimuthal direction $\varphi$ are singled out by joining the pixels hit in the LEID and in the HEID. The accepted events have to be scattered in the hollow cone defined by $\theta_{\min }$ and $\theta_{\max }$, with $\theta_{\min } \in\left[0, \theta_{\max }\right)$ and $\theta_{\max }<\arctan \frac{l}{2 d}$.

a Minimum Detectable Polarization (MDP) and, by definition, only a detection greater than MDP is statistically significant (Weisskopf et al. 2010).

The MDP is calculated from the source and the background fluxes in the selected energy range, $F$ and $B$ respectively, and for the $99 \%$ confidence level it is (Weisskopf et al. 2010):

$$
\mathrm{MDP}=\frac{4.29}{\epsilon \mu F} \sqrt{\frac{B+\epsilon F}{S T}},
$$

where $T$ is the observation time, $S$ the collecting area, $\epsilon$ is the detector efficiency and $\mu$ the modulation factor. This latter parameter is defined as the amplitude of the modulation curve when completely polarized and monochromatic photons are incident on the instrument:

$$
\mu=\frac{M_{\max }-M_{\min }}{M_{\max }+M_{\min }}
$$

where $M_{\max }$ and $M_{\min }$ are the maximum and the minimum of the modulation curve, respectively. A higher value of $\mu$ means that the instrument responds to polarized radiation with a larger modulation and the effect of statistical fluctuations is, in proportion, lower.

In our toy model, we assumed that spurious double events and background are negligible and, therefore, the MDP is inversely proportional to the quality factor $q=$ $\mu \sqrt{\epsilon}$ :

$$
\mathrm{MDP} \simeq \frac{4.29}{\mu \sqrt{\epsilon}} \sqrt{\frac{1}{F S T}} \propto \frac{1}{q} .
$$

In the following we will use the quality factor to optimize the sensitivity of the polarimeter: the larger $q$ the higher the sensitivity. To evaluate $q$, we need to calculate $\mu$ and $\epsilon$ separately.

\subsection{Estimate of the modulation factor}

An estimate of the modulation factor can be computed from some very basic considerations, taking into account the geometry of the instrument and the angular dependence of the differential cross section of Compton scattering. The latter, for completely polarized photons incident on a free electron at rest, is (Klein \& Nishina 1929; Heitler 1954):

$$
\frac{\mathrm{d} \sigma_{K N}}{\mathrm{~d} \Omega}=\frac{1}{2} r_{0}^{2} \frac{E^{\prime 2}}{E^{2}}\left[\frac{E}{E^{\prime}}+\frac{E^{\prime}}{E}-2 \sin ^{2} \theta \cos ^{2} \phi\right],
$$

where $\theta$ is the scattering angle, $\phi$ is defined as the azimuthal angle from the scattering direction to the polarization vector of the incident photon and $r_{0}=\frac{1}{4 \pi \epsilon_{0}} \frac{e^{2}}{m c^{2}}$ is the classical electron radius. The ratio between the energy before and after the scattering $E / E^{\prime}$ is related to the scattering angle $\theta$ by the Compton formula:

$$
\frac{E}{E^{\prime}}=1+\varepsilon(1-\cos \theta)
$$

where $\varepsilon$ is the energy of the incident photon in unit of electron mass, $\varepsilon=E / m_{e} c^{2}$. Equation 4, by substituting Equation 5, becomes:

$$
\begin{aligned}
\frac{\mathrm{d} \sigma_{K N}}{\mathrm{~d} \Omega} & =\frac{1}{2} r_{0}^{2}\left\{\frac{1}{1+\varepsilon(1-\cos \theta)}+\right. \\
& \left.+\frac{1}{[1+\varepsilon(1-\cos \theta)]^{3}}-\frac{2 \sin ^{2} \theta \cos ^{2} \phi}{[1+\varepsilon(1-\cos \theta)]^{2}}\right\}
\end{aligned}
$$


In the following we will be interested only in the angular dependence of the differential cross section. Therefore we define the function $D(\varepsilon, \theta, \phi)$ as:

$$
\begin{aligned}
D(\varepsilon, \theta, \phi) & =\left\{\frac{1}{1+\varepsilon(1-\cos \theta)}+\right. \\
& +\frac{1}{[1+\varepsilon(1-\cos \theta)]^{3}}- \\
& \left.-\frac{2 \sin ^{2} \theta \cos ^{2} \phi}{[1+\varepsilon(1-\cos \theta)]^{2}}\right\} .
\end{aligned}
$$

The first step to evaluate the modulation factor is to calculate the modulation curve, i.e. to make an histogram of the azimuthal scattering directions for completely polarized and monochromatic photons. We have to count how many photons are scattered in a certain azimuthal direction $\phi$, regardless of the scattering angle $\theta$. The number of photons $\mathrm{d} N(\varepsilon, \theta, \phi)$ scattered in the $\phi$ and $\theta$ direction per unit of solid angle $\mathrm{d} \Omega$ at energy $\varepsilon$ is proportional to the differential cross section:

$$
\mathrm{d} N(\varepsilon, \theta, \phi)=\kappa D(\varepsilon, \theta, \phi) \sin \theta \mathrm{d} \theta \mathrm{d} \phi,
$$

where $\kappa$ is a constant of proportionality and $\mathrm{d} \Omega=$ $\sin \theta \mathrm{d} \theta \mathrm{d} \phi$. Neglecting the probability of multiple scatterings in the LEID, the direction measured by joining the hit pixels in the LEID and in the HEID will be that of scattering, and therefore the modulation curve is obtained by summing $\mathrm{d} N / \mathrm{d} \phi$ over the range of $\theta$ values:

$$
\begin{aligned}
M\left(\varepsilon, \varphi, \theta_{\min }, \theta_{\max }\right) & =\int_{\theta_{\min }}^{\theta_{\max }} \frac{\mathrm{d} N(\varepsilon, \theta, \phi)}{\mathrm{d} \phi}= \\
& =\kappa \int_{\theta_{\min }}^{\theta_{\max }} D(\varepsilon, \theta, \phi) \sin \theta \mathrm{d} \theta .
\end{aligned}
$$

The limits of integration, i.e. $\theta_{\min }$ and $\theta_{\max }$, are at large fixed by the geometry of the system. Referring to Figure 1, and using the parameters in Table 1, we have that in our case:

$$
\theta_{\max }=\arctan \frac{l}{2 d} \approx 52^{\circ}
$$

where $l$ is the side of the HEID and $d$ is the LEID-HEID distance, and $\theta_{\min } \in\left[0, \theta_{\max }\right)$. We will discuss in Section 3.3 how more stringent limits on $\theta$ affect the sensitivity to polarization.

In the left hand side of Equation 9 we used the variable $\varphi$ instead of $\phi$ because the latter is the angle to the photon polarization vector, the former is the angle to some axis of reference of the instrument. The two angles are related by

$$
\phi=\varphi-\varphi_{0}+\frac{\pi}{2}
$$

where $\varphi_{0}$ is the angle of polarization defined as the peak of the modulation curve. In the following we will assume $\varphi_{0}=0$ and therefore the maximum of the modulation curve will be at $\varphi=0$ (or $\phi=\pi / 2$ ) and the minimum will be at $\varphi=\pi / 2($ or $\phi=0)$. The modulation factor is eventually derived by applying Equation 2:

$$
\begin{aligned}
& \mu\left(\varepsilon, \theta_{\min }, \theta_{\max }\right)=\frac{M_{\max }-M_{\min }}{M_{\max }+M_{\min }}= \\
= & \frac{M\left(\varepsilon, \varphi=0, \theta_{\min }, \theta_{\max }\right)-M\left(\varepsilon, \varphi=\pi / 2, \theta_{\min }, \theta_{\max }\right)}{M\left(\varepsilon, \varphi=0, \theta_{\min }, \theta_{\max }\right)+M\left(\varepsilon, \varphi=\pi / 2, \theta_{\min }, \theta_{\max }\right)}= \\
= & \int_{\theta_{\min }}^{\theta_{\max }} \frac{D(\varepsilon, \theta, \pi / 2)-D(\varepsilon, \theta, 0)}{D(\varepsilon, \theta, \pi / 2)+D(\varepsilon, \theta, 0)} \sin \theta \mathrm{d} \theta .
\end{aligned}
$$

Equation 12 can be integrated employing standard techniques, but the resulting explicit expression is rather cumbersome and not easy to handle. Consequently, we study the behavior of $\mu\left(\varepsilon, \theta_{\min }, \theta_{\max }\right)$ numerically, by means of the Computer Algebra System MAximA ${ }^{1}$. In Figure 2 we report the modulation factor as a function of $\theta_{\min }$ and $\theta_{\max }$. As an example, we set the energy of incident photons at $30 \mathrm{keV}$ but the qualitative behavior is fully representative, since the dependence of $\mu$ on the energy is rather weak in the range of our interest. The value of the modulation factor increases monotonically with $\theta_{\max }$ and $\theta_{\min }$ and, in principle, the maximum would reach nearly $100 \%$ for $\theta_{\min }, \theta_{\max } \rightarrow 90^{\circ}$. However, this result is not relevant here because, if the scattering angle is close to $90^{\circ}$, the assumption of a single scattering in the LEID (which led to Equation 9) fails and so does our model. Moreover, any feasible layout of the polarimeter discussed in this paper would require $\theta_{\min }$ and $\theta_{\max }$ to be constrained well below $90^{\circ}$. We limited the range of $\theta_{\max }$ and $\theta_{\min }$ between 0 and $70^{\circ}$ in Figure 2 to stress that our results are not applicable for $\theta_{\min }, \theta_{\max } \rightarrow 90^{\circ}$.
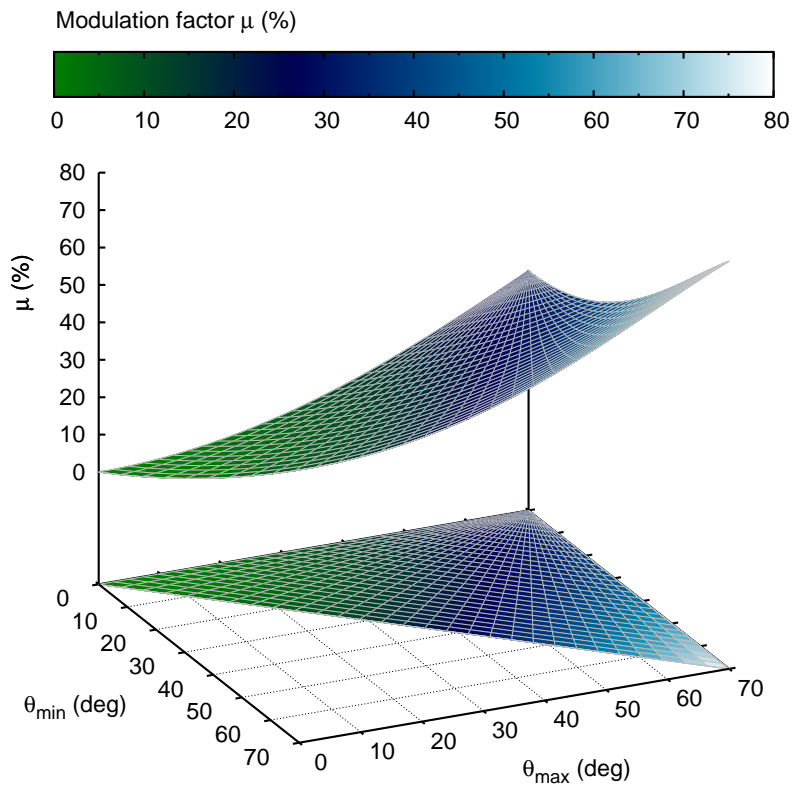

Figure 2. Behavior of the modulation factor as a function of $\theta_{\min }$ and $\theta_{\max }$. The energy of the incident photons is $30 \mathrm{keV}$, but, within the energy range of our interest $(20-100 \mathrm{keV})$, this does not affect the result significantly. We assumed that $0 \leq \theta_{\min }<\theta_{\max }<70^{\circ}$ to stress that our results are not applicable for $\theta_{\min }, \theta_{\max } \rightarrow 90^{\circ}$.

\subsection{Estimate of the efficiency}

1 http://maxima.sourceforge.net/ 
The efficiency $\epsilon$ of detecting scattered photons has to take into account three main contributions: $(i)$ the probability of Compton scattering in the LEID, (ii) the photoabsorption efficiency in the HEID and (iii) that only a fraction of photons are actually scattered towards the HEID. We evaluate the first term, $\epsilon_{\text {scatt }}^{L}(E)$, as the fraction of Compton scatterings among all of the interactions which occur in the LEID:

$$
\epsilon_{\text {scatt }}^{L}\left(E, x_{L}\right)=\frac{\mu_{\text {scatt }}^{L}(E)}{\mu_{\text {tot }}^{L}(E)}\left[1-\exp \left(-\mu_{\text {tot }}^{L}(E) \rho^{L} x^{L}\right)\right]
$$

where $\mu_{\text {tot }}^{L}$ and $\mu_{\text {scatt }}^{L}$ are the total and the incoherent scattering attenuation coefficients for the LEID, $\rho^{L}$ is the density of the LEID material (Silicon, in our model) and $x^{L}$ its thickness.

The second contribution to the efficiency takes into account the probability that the scattered photon is absorbed in the HEID. We will assume in the following that the photoabsorption efficiency of the HEID is 100\% in the energy range of our interest, i.e.:

$$
\epsilon_{\mathrm{abs}}^{H}=1 .
$$

This assumption, although naive, will not affect our results significantly. In effect, it is quite reasonable because the requirement for the HEID to have an high efficiency in the entire energy range is a heritage of its use as an imager. In the configuration assumed (see the Table 1) the $x^{H}=2 \mathrm{~mm}$ thickness allows for the photoelectric absorption of more that $80 \%$ of $100 \mathrm{keV}$ photons incident on-axis. Also, the efficiency in the polarimetric mode is actually higher, because the scattered photons see the detector as having an effective depth $x^{H} / \cos \theta$. Claiming a $100 \%$ HEID efficiency allows both to simplify the following discussion and to derive the maximum possible sensitivity to polarization.

The last contribution to the efficiency is due to the fact that, while photons are scattered over all the solid angle, only in a few cases their scattering direction crosses the HEID. Actually, we set a more stringent limit, i.e. we take only those photons scattered at angles between $\theta_{\min }$ and $\theta_{\max }$, no matter the value of the azimuthal angle $\phi$. The fraction of accepted photons $\epsilon_{c}$ is calculated integrating $\mathrm{d} N(\varepsilon, \theta, \phi)$ (see Equation 8):

$$
\begin{aligned}
& \epsilon_{\mathrm{c}}\left(\varepsilon, \theta_{\min }, \theta_{\max }\right)=\frac{\int_{0}^{2 \pi} \int_{\theta_{\min }}^{\theta_{\max }} \mathrm{d} N(\varepsilon, \theta, \phi)}{\int_{0}^{2 \pi} \int_{0}^{\pi} \mathrm{d} N(\varepsilon, \theta, \phi)}= \\
& =\frac{\int_{0}^{2 \pi} \int_{\theta_{\min }}^{\theta_{\max }} D(\varepsilon, \theta, \phi) \sin \theta \mathrm{d} \theta \mathrm{d} \phi}{\int_{0}^{2 \pi} \int_{0}^{\pi} D(\varepsilon, \theta, \phi) \sin \theta \mathrm{d} \theta \mathrm{d} \phi} .
\end{aligned}
$$

uated from the three aforementioned contributions:

$$
\begin{aligned}
& \epsilon\left(\varepsilon, \theta_{\min }, \theta_{\max }, x^{L}\right)= \\
= & \epsilon_{\text {scatt }}^{L}\left(\varepsilon, x_{L}\right) \times \epsilon_{\mathrm{abs}}^{H} \times \epsilon_{\mathrm{c}}\left(\varepsilon, \theta_{\min }, \theta_{\max }\right)= \\
= & \frac{\mu_{\mathrm{scatt}}^{L}(\varepsilon)}{\mu_{\mathrm{tot}}^{L}(\varepsilon)}\left[1-\exp \left(-\mu_{\mathrm{tot}}^{L}(\varepsilon) \rho^{L} x^{L}\right)\right] \times \\
\times & \frac{\int_{0}^{2 \pi} \int_{\theta_{\min }}^{\theta_{\max }} D(\varepsilon, \theta, \phi) \sin \theta \mathrm{d} \theta \mathrm{d} \phi}{\int_{0}^{2 \pi} \int_{0}^{\pi} D(\varepsilon, \theta, \phi) \sin \theta \mathrm{d} \theta \mathrm{d} \phi},
\end{aligned}
$$

where we used $\varepsilon=E / m_{e} c^{2}$ to express the dependence on the energy of the incident photon.

As for the modulation factor, we likewise plot in Figure 3 the behavior of $\epsilon\left(\varepsilon, \theta_{\min }, \theta_{\max }\right)$ as a function of $\theta_{\min }$ and $\theta_{\max }$ for $30 \mathrm{keV}$ photons. The qualitative result is rather simple: the efficiency is higher when the interval of accepted scattering angles is larger $\left(\theta_{\max } \rightarrow 90^{\circ}\right.$ and $\left.\theta_{\min } \rightarrow 0\right)$. More remarkably, the absolute value of the efficiency is always lower that $1 \%$. As a matter of fact, the value of $\epsilon_{\text {scatt }}^{L}$ is $1.6 \%$ for the assumed thickness of the LEID $(450 \mu \mathrm{m})$ at $30 \mathrm{keV}$ and the incomplete collection of the scattered photons makes things worse. The low intrinsic efficiency is the most important deficiency in the design of a polarimeter based on stacked imaging detectors and it cannot be easily overcome. The only way is to increase the thickness of the depleted region of the LEID, but the value of $\epsilon_{\mathrm{scat}}^{L}$ is only $3.4 \%$ still in the case of a depleted region $1 \mathrm{~mm}$ thick, which is one of the largest values still technologically reasonable. Moreover the increase of the thickness would result in a larger background and would spoil the performance of the LEID for the detection of faint sources.

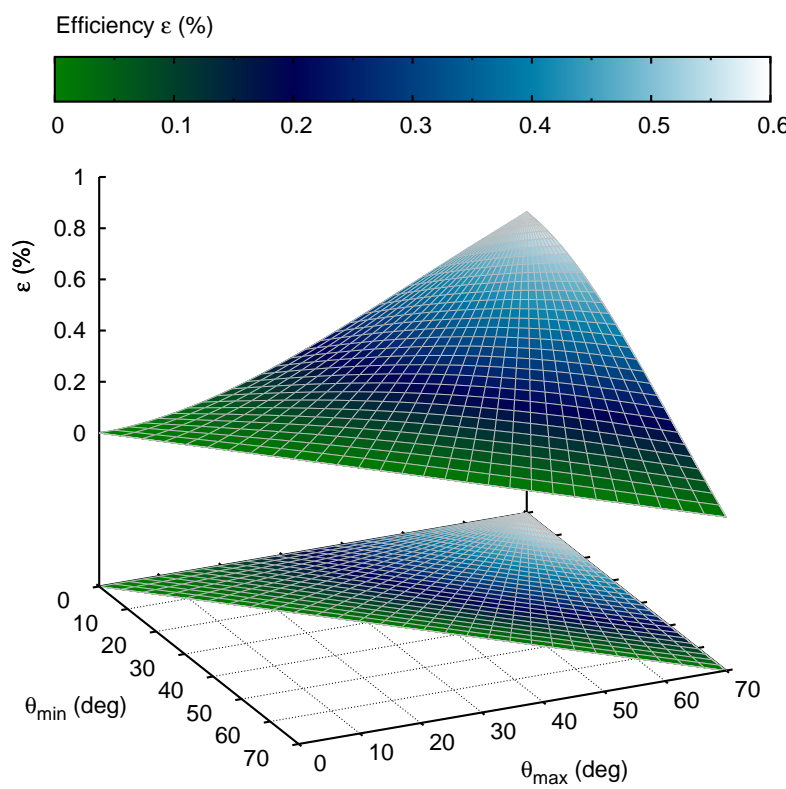

Figure 3. Behavior of the efficiency as a function of $\theta_{\min }$ and $\theta_{\max }$ for a LEID thickness of $450 \mu \mathrm{m}$. The energy of the incident photons is $30 \mathrm{keV}$, but, within the energy range of our interest (20-100 keV), this does not affect the result significantly. 


\subsection{Quality factor}

Equations 12 and 16 can be combined to obtain the quality factor $q=\mu \sqrt{\epsilon}$. Notably, we enclosed the dependence of $q$ on the geometry of the detector in only three parameters, that are $\theta_{\min }, \theta_{\max }$ and $x^{L}$. This allows us to optimize the sensitivity of the polarimeter varying a very limited number of free quantities.

The behavior of the quality factor at $30 \mathrm{keV}$ with respect to $\theta_{\min }$ and $\theta_{\max }$ is reported in Figure 4 for $x^{L}=450 \mu \mathrm{m}$. As expected, the sensitivity monotonically increases with $\theta_{\max }$ because both modulation factor and efficiency do. In the geometry assumed in Table 1, $\theta_{\max } \lesssim 52^{\circ}$ (cf. Equation 10) and therefore we freeze $\theta_{\max }$ to $52^{\circ}$ in order to maximize $q$. On the contrary, the dependence on $\theta_{\min }$ is more complex. The interplay between the increasing modulation factor and the decreasing efficiency originate a broad peak in the quality factor. This unexpected result is shown more clearly in Figure 4 where the dependence of $q$ with respect to $\theta_{\min }$ and energy is reported for $\theta_{\max }=52^{\circ}$. The peak is basically independent on energy in the range on our interest, but depends on $\theta_{\max }$. A practical relation which linkes $\theta_{\max }$ and the value of $\theta_{\min }$ which maximizes $q$ is:

$$
\theta_{\min }^{\text {peak }}[\mathrm{deg}] \approx 0.57 \theta_{\max }[\mathrm{deg}] .
$$

In our case, $\theta_{\max }=52^{\circ}$ and therefore $\theta_{\min }^{\text {peak }} \approx 30^{\circ}$. We will use these values in the following as those which provide the best sensitivity to polarization, but the choice to accept only events scattered with an angle larger than a certain threshold has also practical advantages. Events scattered at angles lower than $30^{\circ}$ release in the LEID less than $0.23 \mathrm{keV}$ at $30 \mathrm{keV}$ according to the Compton formula (see Equation 5), and such a low energy deposit may be difficult to detect.

\section{MONTE CARLO SIMULATIONS}

The results discussed in the previous section are purely analytical. Moreover, they rely on some simplified physical assumptions, e.g. in treating the Compton scattering as from free electrons at rest. In the general case of the scattering inside a realistic material, however, the momentum distribution of the electrons and the binding effects in atoms will produce both a broadening of the Compton peak in the scattered photon spectrum and a suppression of the scattering at forward angles (Veigele \& Tracy 1966; Bergstrom \& Pratt 1997; Hubbell 1997). The incoherent scattering cross section thus becomes:

$$
\frac{\mathrm{d} \sigma_{\text {inc }}}{\mathrm{d} \Omega}=S_{F}(x, Z) \frac{\mathrm{d} \sigma_{\mathrm{KN}}}{\mathrm{d} \Omega},
$$

where $S_{F}(x, Z)$ is the so-called scattering function and depends on the transferred momentum $x \approx$ $(E / h c) \sin (\theta / 2)$ and on the atomic number $Z$ of the scattering atom. The efficiency for a given scattering polar angle $\theta$ is therefore affected. The azimuthal angle $\phi$, on the contrary, can be assumed independent of the binding effects (Matt et al. 1996). Another effect to take into account is due to the "pixelization" of the detectors, and to the geometrical shape of the pixel itself. To make a meaningful comparison with a realistic case, we therefore developed a Monte Carlo simulator of our toy model for a stacked polarimeter (see Table 1), using the Geant4 toolkit (Agostinelli et al. 2003), version 4.9.4. Accurate physics lists were employed for the various electromagnetic interactions, notably the Livermore low energy libraries $^{2}$ that use the EPDL97 tabulated version of the scattering function (Hubbell et al. 1975).

Geant4 simulations allow us to treat the physics of the scattering more accurately. Notwithstanding, we intentionally maintain also in Monte Carlo treatment some simplified assumptions in the design of the instrument. In particular, we do not apply any energy threshold to the event detection in the LEID and consider LEID and HEID as having an infinite energy resolution. We are aware that these parameters play an important role in the determination of a good estimate of the detection efficiency, especially at low energy. In particular, any realistic assumption on the energy threshold and on the spectral resolution would decrease the efficiency and hence the sensitivity to polarization. Nonetheless, this choice is in line with our primary goal, that is to argue on the intrinsic sensitivity to polarization of the stacked imager layout rather than to propose a feasible design and to discuss its sensitivity.

\subsection{Data Analysis}

For each event (i.e. for each primary photon generated in the simulation run), the output of the Geant4 Monte Carlo simulator is a list of the pixels having a non-zero energy deposit, with their location and deposited energy. Only double events which release an amount of energy simultaneously in both the LEID and the HEID are relevant to study polarization and are recorded. However, among them we must select the good events, which are those for which it is possible to reconstruct the direction of scattering, and therefore are useful to measure the polarization of the incident photon beam. Examples of double but not good events are those in which one of the detectors collects the fluorescence radiation emitted after the photoabsorption in the other detector or events which undergo multiple scatterings in different pixels, especially in the LEID. Another process which also produces double events is the photoabsorption in the few microns at the bottom surface of the LEID: in this case the photoelectron may escape from the LEID and it could be absorbed in the HEID. These events, albeit rare, must be removed because the direction of emission of the photoelectron is sensitive to polarization but the effect is opposite to that of the Compton scattering, i.e. the probability of emission is maximum along the polarization. In our simulations we used a $30 \mu \mathrm{m}$ thick beryllium plate between the LEID and the HEID to stop the large part of the photoelectrons but not X-rays (the transparency of the plate at $20 \mathrm{keV}$ is $99.9 \%$ on-axis). However the configuration of such a shield could be conveniently adapted to specific needs in actual instruments, for example it could be a thin thermal shield if the LEID and the HEID must work at different temperatures.

We defined some criteria to filter those events in which the photon underwent only two interactions, the first as a scattering in the LEID and the second in the HEID, restricting ourselves only to procedures which could be applied also to data collected by real instruments. The first filter is a spatial filter, and it is based on the fact

2 https://twiki.cern.ch/twiki/bin/view/Geant4/ LoweMigratedLivermore 


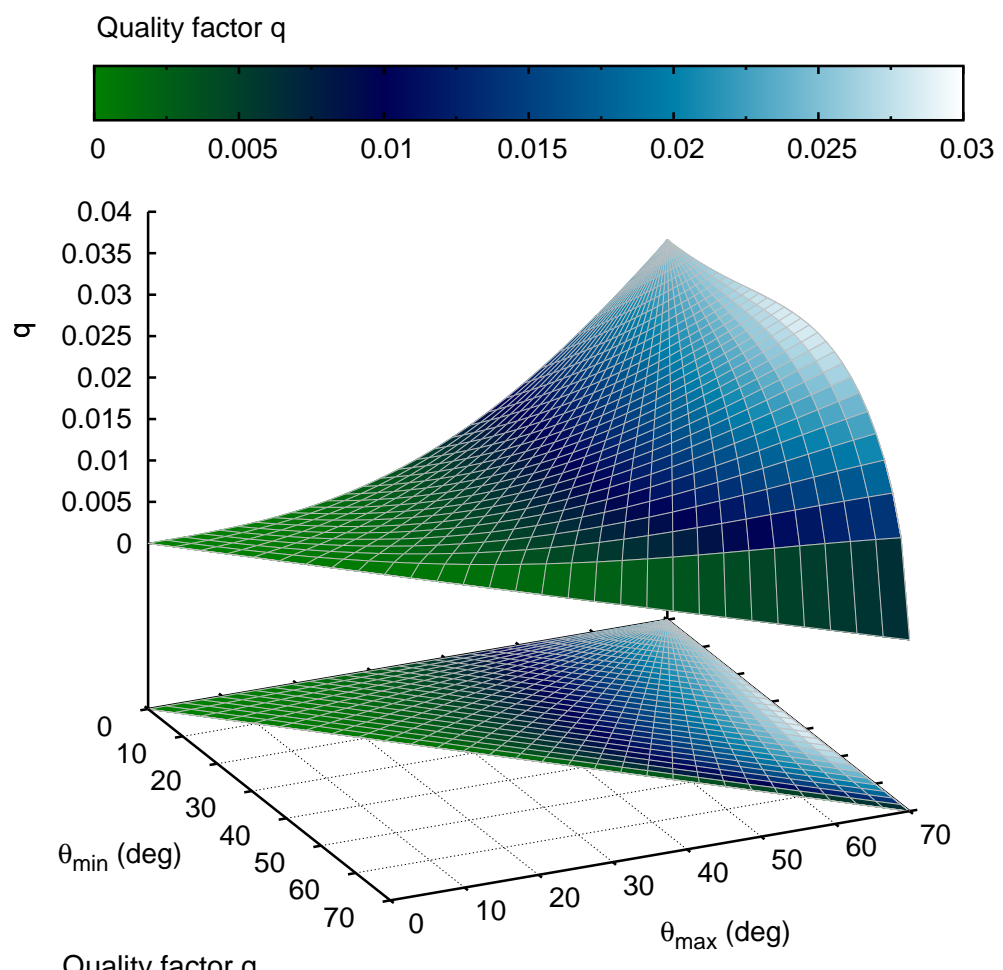

Quality factor q

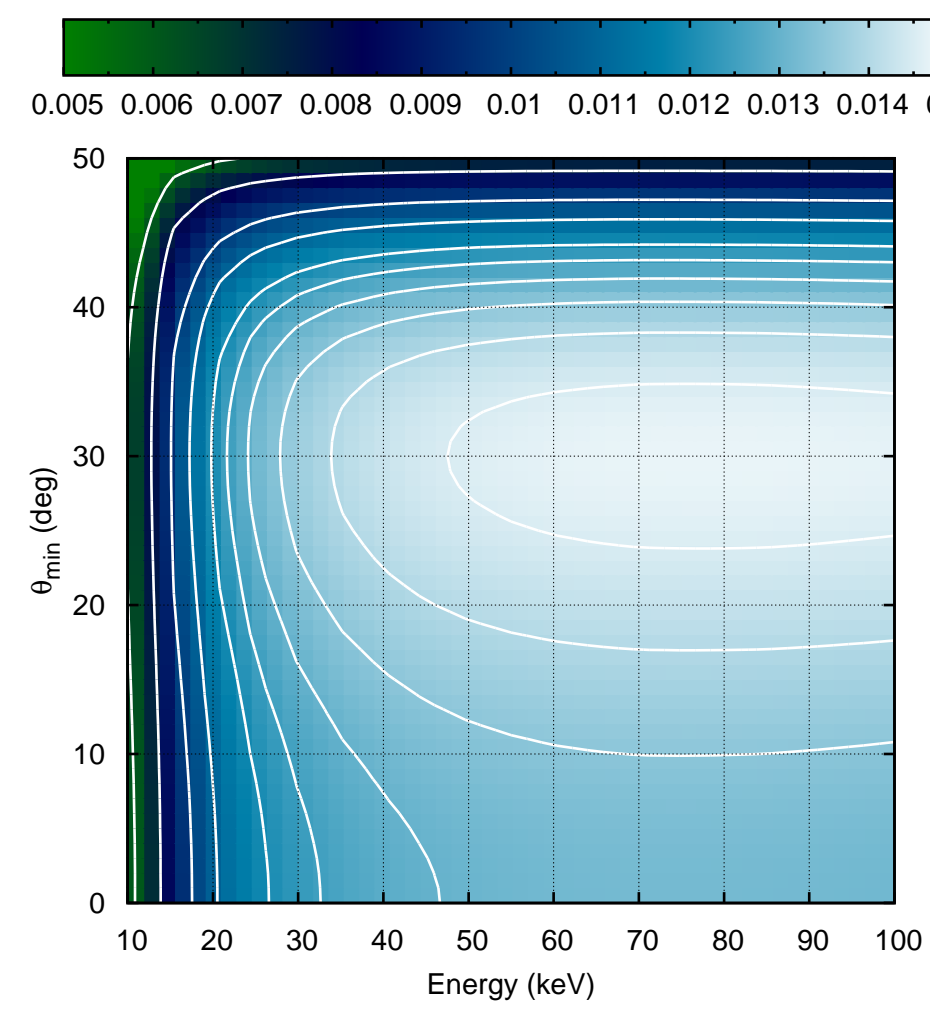

Figure 4. (a) Behavior of the quality factor as a function of $\theta_{\min }$ and $\theta_{\max }$ at $30 \mathrm{keV}$. (b) Quality factor as a function of $\theta_{\min }$ and energy for $\theta_{\max }=52^{\circ}$ 
that good events must undergo the first LEID scatter in the same pixels in which the source is imaged. An accurate model of the instrument should consider that the image of the source is distributed on the focal plane according to the point spread function of the telescope. Instead, in our toy model we just choose a circular beam distributed uniformly in the four central pixels of the detector. Therefore we select only those events which release at least an energy deposit in one of these pixels and/or in their adjacent ones. Secondly, we imposed a criterion on the number of hit pixels (hit pixels filter). We select only those events for which there is only one energy deposit in the LEID, which must be in a location compatible with the spatial filter, and only one hit pixel in the HEID. The second condition must be applied with some attention because we experienced that the charge produced by a photoabsorption event may be spread over a cluster of several non-contiguous pixels, mainly because fluorescence photons may be absorbed at some distance from the photoabsorption point. We treat such a cluster as a unique pixel with a charge equal to the total energy deposit and a position weighted with the energy collected in each pixel of the cluster. Operatively, we consider that all pixels less distant than 10 pixels belong to the same cluster. Although such a large summation radius may imply some drawbacks on a real device, especially to remove the particle background, large clusters must be correctly included in the analysis to avoid systematics on the modulation curve due to the square pattern of the pixels (see Figure 8). A third filter is applied on the energy collected on the HEID, which must be higher than $5 \mathrm{keV}$ (HEID energy filter). Such a filter has not a large impact on the efficiency $(\sim 3 \%$ at $30 \mathrm{keV})$ or on the modulation factor (variation below the statistical error). Notwithstanding, we imposed a energy threshold for the HEID events in order to remove those double events in which the photon is absorbed in the LEID and the fluorescence of Silicon at $\sim 1.7 \mathrm{keV}$ in the HEID. The $5 \mathrm{keV}$ value is reasonable for current CdTe detectors. The last filter we defined, called $\theta$-filter, is a filter on the scattering angle, measured by the position of the pixels hit in the two detectors. We used the same limits derived in Section 3.3, that is we restricted the scattering angle between 30 and $52^{\circ}$.

The spectrum obtained for the LEID, the HEID and by summing event by event the energy deposits in the two detection planes is shown in Figure 5 for $30 \mathrm{keV}$ incident photons. The spectrum including all events is reported as a gray histogram, while that obtained after applying the filters discussed above is in black. A lot of prominent lines are visible and correspond to the escape peaks or to the absorption of fluorescence lines from Silicon (Klines at $\sim 1.7 \mathrm{keV}$ ), Cadmium (K-lines at $\sim 23-26 \mathrm{keV}, \mathrm{L}$ lines at $\sim 3 \mathrm{keV}$ ) or Tellurium (K-lines at $\sim 27-31 \mathrm{keV}$, only visible for photons above $\sim 32 \mathrm{keV}$, and $\mathrm{L}$ lines at $\sim 4 \mathrm{keV})$. Good events are basically characterized by a small energy deposit $E_{L}$ in the LEID (a few keV) and by an energy deposit in the HEID equal to $E-E_{L}$, where $E$ is the energy of the incident photons. These events fill in the total spectrum the energy bin around $30 \mathrm{keV}$ and represent by far the main components after applying the filters (black histogram in Figure 5). Another class of good events are those which deposit in the HEID an energy equal to $E-E_{L}$ but the fluorescence photon, which is emitted with high probability ( $>80 \%$ for K-shell photoabsorption and high-Z material like Cadmium and Tellurium), escapes from the detector. In this case the energy detected in the HEID is $E-E_{L}-E_{f}$, where $E_{f}$ is the energy of the fluorescence photon, and therefore these events make up an escape peak in the total spectrum at energy $E-E_{f}$. In Figure 5 two of such escape peaks are visible at about $7 \mathrm{keV}$, which correspond to the escape of $\mathrm{Cd} \mathrm{K}_{\alpha 1}$ and $\mathrm{Cd} \mathrm{K}_{\alpha 2}$ fluorescence photons with energy $23.2 \mathrm{keV}$ and $23.0 \mathrm{keV}$, respectively. It is worth mentioning that the application of the $\theta$-filter has the positive effect to make our results less dependent on the energy threshold of the LEID. As a matter of fact, the removal of events scattered at less of $30^{\circ}$ cut out the large part of the events which deposit in the LEID only a few hundreds of $\mathrm{eV}$ (see the inset in top panel of Figure 5). For the sake of completeness, we also show in Figure 6 the spectrum for $90 \mathrm{keV}$ incident photons. The main features remain the same as those discussed in the case of $30 \mathrm{keV}$ radiation, although in this case it is visible a low energy bump in the HEID and in the total spectrum which corresponds to photons which, after the scattering in the LEID, scatter a second time in the HEID. Such events are still good to measure polarization, although their statistical weight is negligible with respect to the events which are absorbed after the first scattering.

Events which pass all the filters described above are used to build a modulation curve which is fitted with the function

$$
\mathcal{M}(\varphi)=A+B \cdot \cos \left(\varphi-\varphi_{0}\right) .
$$

The modulation factor $\mu$ is derived as usual by (cfr. Equation 2)

$$
\mu=\frac{\mathcal{M}_{\max }-\mathcal{M}_{\min }}{\mathcal{M}_{\max }+\mathcal{M}_{\min }}=\frac{B}{B+2 A} .
$$

\subsection{Results}

We performed simulations at 20, 30, 40, 50, 60, 70, 80, $90 \mathrm{keV}$ and $100 \mathrm{keV}$, generating $50 \cdot 10^{6}$ photons for each energy. Taking as an example the simulation at $30 \mathrm{keV}$, we detected $1.2 \cdot 10^{6}$ double events, that are for the large part events in which the photon is absorbed in the HEID and some fluorescence in the LEID. Among them, 162 . $10^{3}$ events were selected by the spatial filter, $156 \cdot 10^{3}$ passed both the spatial and the hit pixels filter, $151 \cdot 10^{3}$ passed also the HEID energy filter and eventually $93 \cdot 10^{3}$ passed also the $\theta$-filter. We repeated the simulations for three different values of the angle of polarization, that are $0^{\circ}, 25^{\circ}$ and $90^{\circ}$, to check the consistency of our results. In this respect, the first and the last values represent the two extreme conditions, while $25^{\circ}$ is just an intermediate value not in resonance with any expected on a square pattern.

Examples of the typical modulation curves obtained from Monte Carlo are shown in Figure 7 in the case of $30 \mathrm{keV}$ photons and a polarization angle of $0^{\circ}$ or $25^{\circ}$. The fit with the square-cosine function is very good and the reduced $\chi^{2}$ is 1.067 and 1.042 respectively for 97 degrees of freedom. We report in the same Figure as the gray histogram also the modulation as it would appear without applying the $\theta$-filter. In this case a strong systematic effects appears in the modulation curve which is simply due to the fact that the HEID detection plane is 


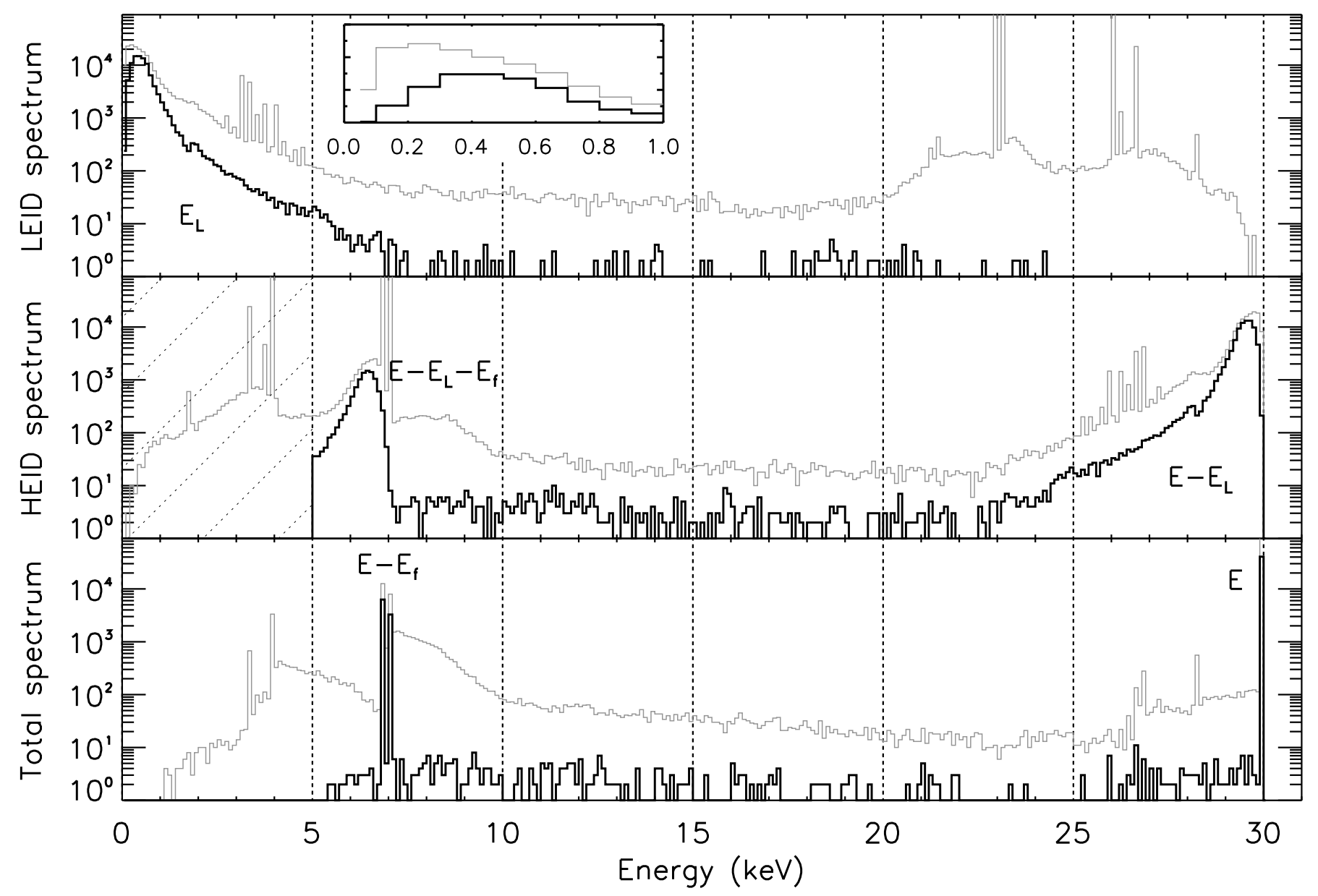

Figure 5. Spectrum measured by the LEID (top) and by the HEID (middle) for $30 \mathrm{keV}$ incident photons. The total spectrum, obtained by summing the energy collected by the two instruments event by event, is in the bottom panel. The inset in the top panel shows a zoom at low energy of the LEID spectrum. In black and gray are reported the spectrum with and without the filters discussed in the text to distinguish real scattering events, including the $\theta$-filter. The diagonally dashed region in the middle panel shows the energy threshold imposed for the HEID with the energy filter.

square. As a matter of fact, in each bin of the modulation curve there is the number of events scattered in a certain azimuthal angular bin regardless the scattering angle. Without the $\theta$-filter, the scattering angle is only constrained by the geometry of the two detection planes because the photon must cross the HEID to be detected. However, the detection planes are square, and this implies that the interval of accepted scattering angles is larger in the diagonal directions thus causing a bump in the number of collected events.

Although we neglected all of the main causes which may induce a systematic modulation for a real instrument, it is interesting to look at the behavior of the modulation curve for completely unpolarized photons. This is reported in Figure 8 for $90 \mathrm{keV}$ photons, in which as above the black and gray histograms are those obtained with or without applying the $\theta$-filter, respectively. As expected, the detected modulation is consistent with zero with a $33 \%$ confidence level. However, it is also interesting to see what would be the result of our analysis in case of slightly different filter parameters. When we described the hit pixels filter in the Section 4.1, we mentioned that a large summation radius, that was 10 pixels, is required to avoid systematic effects on the modulation curve. In the lower panel of Figure 8 we show the modulation curve obtained by exactly the same data as in the upper panel, but with the only difference that the summation radius is 1.6 pixels instead of 10 . In this case only pixels which are contiguous on the side or on the diagonal are considered as a unique cluster and therefore only events involving a single cluster of contiguous pixels pass the hit pixels filter. The result is that a strong systematics due to the square pattern of the pixels emerges with a peak to peak variation of $\sim 10 \%$. Although formally the square-cosine modulation is still consistent with unpolarized radiation at a $15 \%$ confidence level, this is only due to the high symmetry of the four peaks. In actual devices also small nonuniformities would cause a much higher modulation and in the most extreme situation the spurious polarization, obtained by dividing the peak to peak variation by the modulation factor, would be $\sim 10 \% / \mu \approx 30 \%$. As a matter of fact, spurious modulated signals can arise from minor effects even in apparent symmetrical geometries.

In Figure 9, the modulation factor, the efficiency and the quality factor obtained from Monte Carlo are compared to the values derived by the analytical treatment discussed in Section 3. The efficiency is calculated as the ratio between the number of events which passed all filters and that of incident photons. The agreement is in general rather good, although there are some de- 


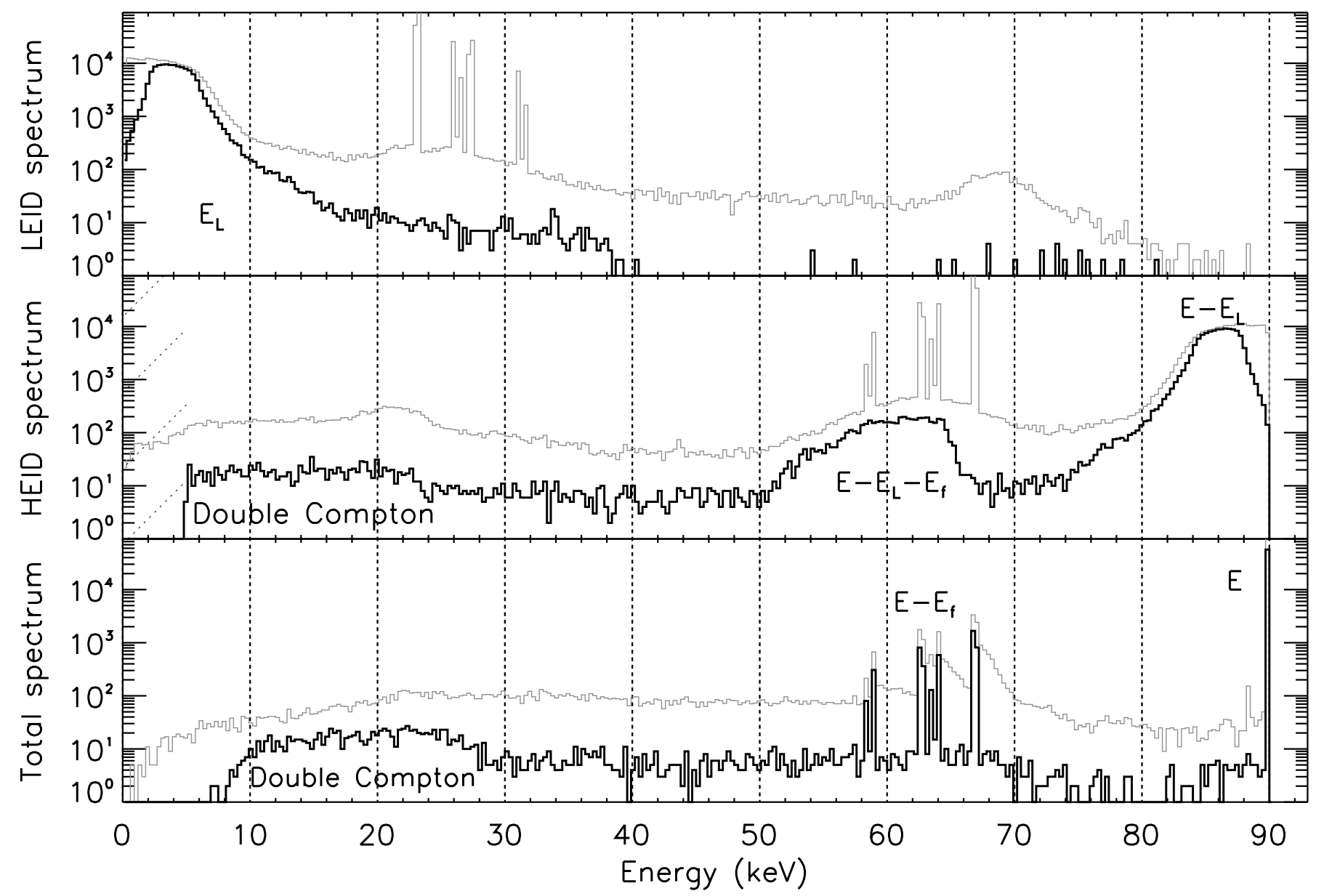

Figure 6. The same as Figure 5 but for $90 \mathrm{keV}$ photons.

viations with respect to the expected dependence. In particular, the modulation factor is larger and the efficiency is smaller than expected, with a difference which decreases with energy. This disagreement is easily explainable in view of the fact that in Monte Carlo simulations we treated Compton scattering more accurately by including the scattering function. As a matter of fact, this has the effect to suppress the forward scattering, especially at low scattering angles and at low energy. Only forward scatterings are collected in the stacked imager layout and therefore an efficiency reduction is a straightforward consequence of introducing the scattering function. This can as well explain the increase of the modulation factor because the suppression is more effective at low scattering angles, where the intrinsic modulation with polarization is lower. This interpretation is confirmed by studying the scattering angle distribution of the events which passed the filters. At low energy (see Figure 10, left panel) there is a significant difference between the distribution obtained from Monte Carlo and that expected from the Klein-Nishina formula, especially at low scattering angles. This explains why the estimates of the modulation factor and of the efficiency obtained analytically and with the Monte Carlo are different. Instead the results are more in agreement at higher energy, where the two distributions differ less (see Figure 10, right panel).

Monte Carlo simulations allow us to confirm another result obtained in the analytical treatment presented in Section 3, that is the maximum of the sensitivity for $\theta_{\min } \approx 30^{\circ}$ (see Equation 17). In Figure 11 we show the quality factor as a function of $\theta_{\min }$ for $\theta_{\max }=52^{\circ}$ and $30 \mathrm{keV}$ photons. The dependence obtained from the simulations is closely related with the expected one, with a small offset which can be again ascribed to the inclusion of the scattering function in the simulations.

\section{CONCLUSIONS}

The primary aim of this work was to assess the intrinsic sensitivity to polarization of the stacked imager design more than to discuss the feasibility and the performance of a particular implementation. In this respect, the results of our analytical treatment and of Monte Carlo Geant4 simulations are in full agreement. The first noteworthy result is the discovery of a peak in the quality factor, a quantity assumed to be representative of the polarization sensitivity, for a particular range of accepted scattering angles. It is worth mentioning that this non-obvious result firstly came out by our analytical treatment. This suggests that an optimization of Compton scattering polarimeters can (and should) be done also with elementary considerations based on the KleinNishina cross section. Unfortunately, our main result is that the stacked imager design has a very low sensitivity to polarization despite our optimistic assumptions. We are far from an optimized layout and some improve- 

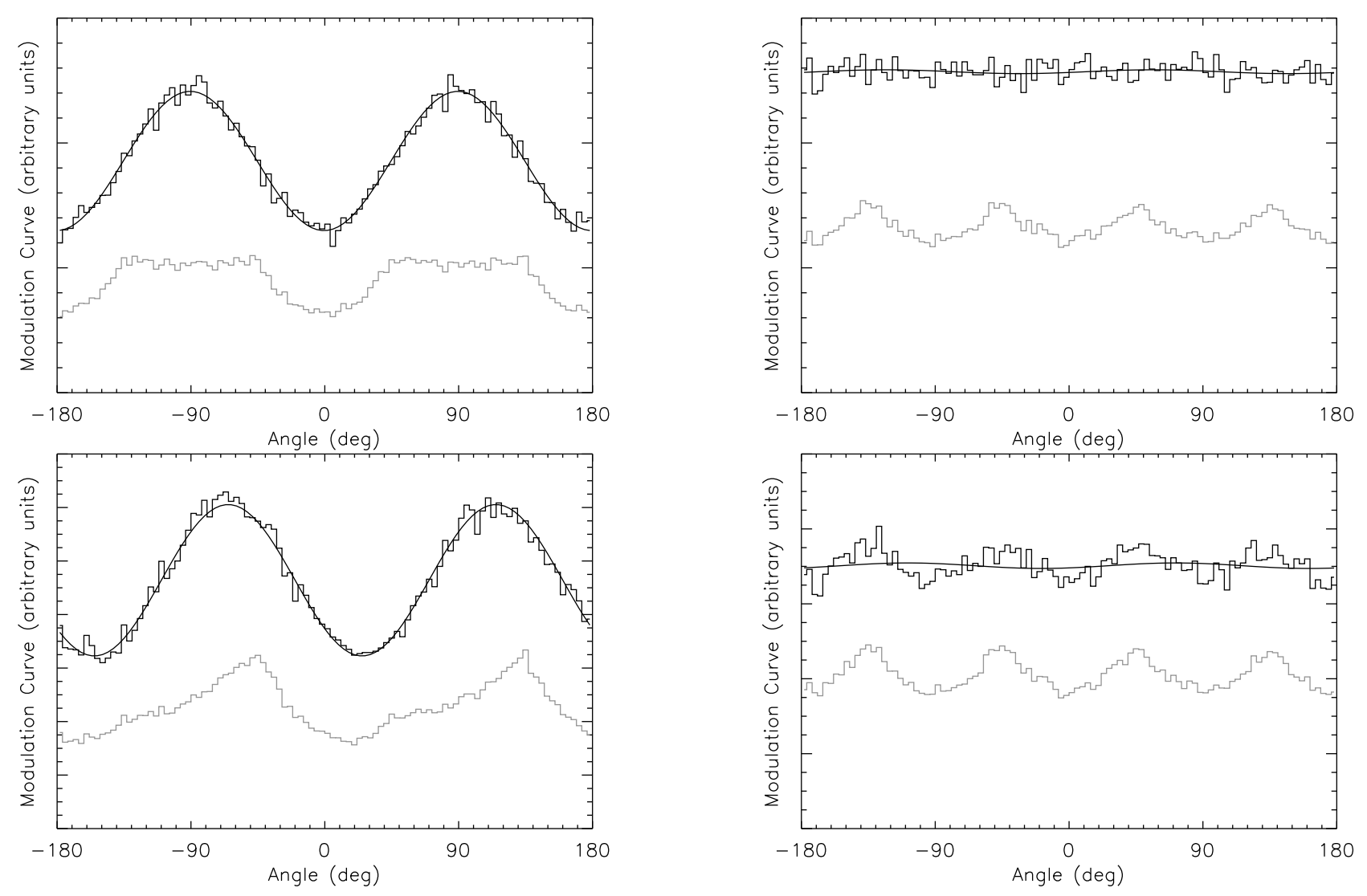

Figure 7. Modulation curve obtained from Monte Carlo simulations for an angle of polarization $0^{\circ}$ (upper panel) or $25^{\circ}$ (lower panel). The black and gray histograms refer to the modulation curve obtained if the $\theta$-filter is applied or not, respectively. The black and gray histograms are not to scale.

ment is still possible with a more refined data analysis. For example, in the stacked imager design the appropriate modulation factor could be associated to each event depending on the scattering angle instead of using an average value. Also, it would be possible to add more detectors encircling the imagers in order to detect photons scattered on a large fraction of the solid angle and not only in the forward direction. However, all of these tricks may improve the sensitivity of a factor of a few at maximum while the low sensitivity of the stacked imager design is largely limited by the very low probability for scattering in the first detection plane. At this regard, we do not think that a major improvement is possible since our estimates are based on the fundamental physics of the Compton scattering. An increase in the scattering efficiency, even though not outstanding, would be possible only by increasing the Silicon thickness. However this would unavoidably worsen the performance of the detector as an imager and this may be unacceptable, since the main use of such a stacked imaging detector would be in any case to image photons on a large energy range. In addition, the dependence of the quality factor on the efficiency is rather weak and, roughly speaking, doubling the efficiency would result in just a $40 \%$ increase in quality factor.

Obviously, a certain polarization sensitivity, albeit low, is present in any case and the common argument is that it can be exploited "for free". However we think that

Figure 8. Modulation curve obtained for unpolarized radiation at $90 \mathrm{keV}$. Upper panel: Modulation curve obtained from Monte Carlo simulations if the $\theta$-filter is applied or not (black and gray histogram respectively). The two histograms are not to scale. Lower panel: The same as the upper panel, but in the case the summation radius is 1.6 instead of 10, that is only contiguous pixels are considered as an unique cluster.

this argument should be discussed with care because the choice to use stacked imagers to detect scattered photons poses some requirements which are usually not set if the instrument is used just as an imager. For example, fast coincidence between the two detection planes is an ad-hoc requirement. Specific calibration campaigns with polarized and unpolarized radiation would be required in order to obtain reliable results and to single out e.g. nonuniformities in the pixel response. Dedicated efforts would also be needed for data analysis. In the most optimistic assumption of negligible background, good events are still overwhelmed by other double events by an order of magnitude, and the application of some of the filters that we used, e.g. the hit pixel filter, may be difficult for real instruments. Another issue which we intentionally disregarded but requires significant efforts when dealing with real instruments is the control of the systematics which may be severe for the stacked imager layout.

The low sensitivity we estimated is also not encouraging when it is compared with the performance of dedicated instruments. For example, the focal plane Compton polarimeter discussed by Soffitta et al. (2010) (but see also Fabiani et al. 2012 in preparation) has a quality factor which is higher than 0.40 at $30 \mathrm{keV}$ and almost constant with energy, i.e. at least a factor $\sim 30$ higher than that of a stacked imager polarimeter. Roughly speaking, this means that the same MDP can be reached 


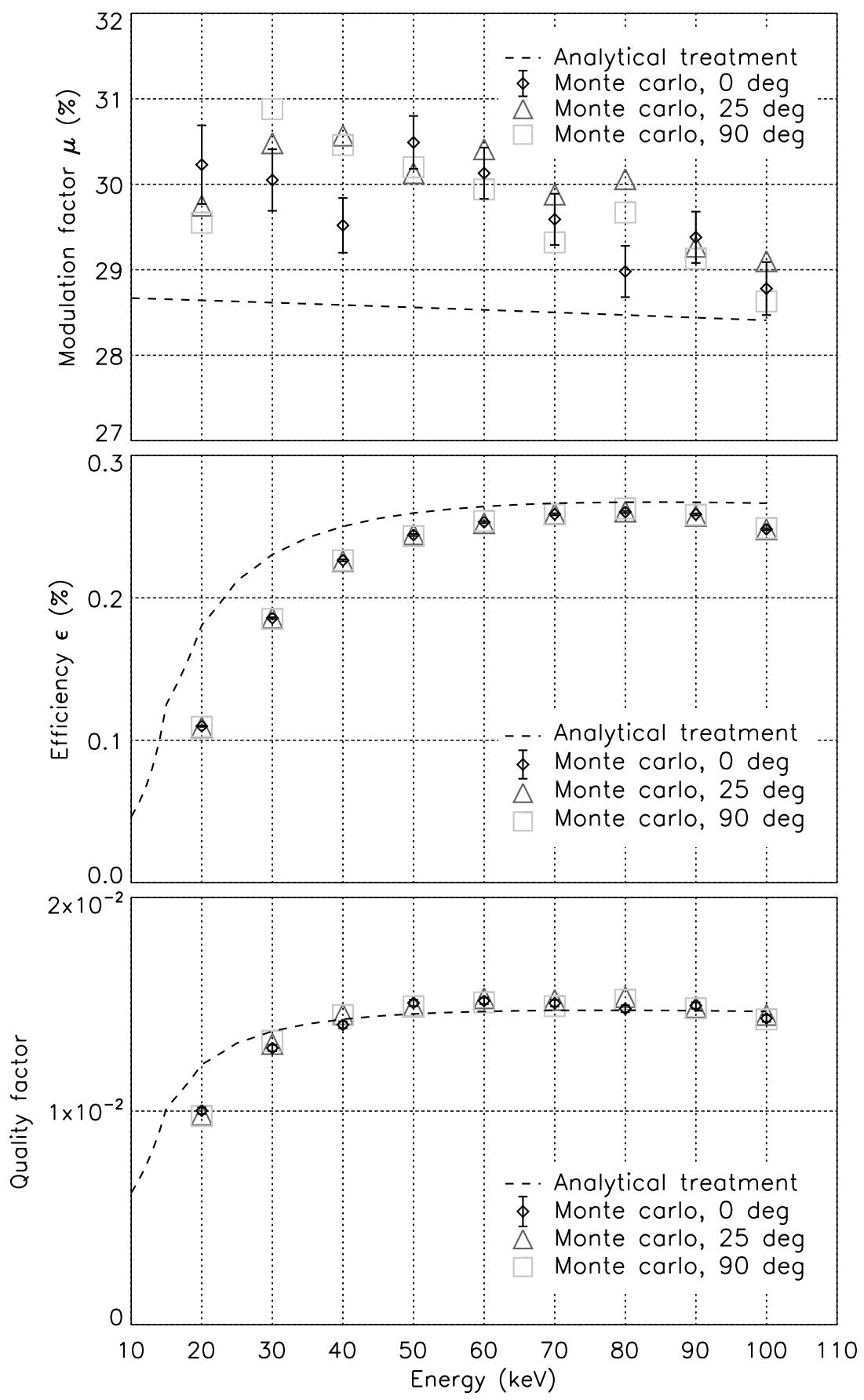

Figure 9. Comparison of the modulation factor (top), efficiency (middle) and quality factor (bottom) as derived by the analytical treatment (dashed line) and by the Monte Carlo simulations (points). The different symbols refer to different values of the incident photons angle of polarization, $0^{\circ}, 25^{\circ}$ or $90^{\circ}$. Error bars are shown only for the first value of the polarization angle for graphical reasons, but they are representative for the other values too. The small disagreement between the analytic and Monte Carlo results, which decreases with energy, is due to the fact that in Monte Carlo simulations we treated more accurately the physics of the Compton scattering.

with an observation $\sim 30^{2}=900$ times shorter. This obviously discourages the efforts to develop and calibrate the polarization capabilities of forthcoming stacked imagers and rather suggests to use dedicated instruments. A possible policy which could be adopted in case of missions with a large telescope is to mount the stacked imager to perform imaging and a dedicated polarimeter on a movable platform and to place alternatively the two instruments in the focus of the telescope. For example, this was the strategy adopted for XEUS/IXO. If the science objectives of the mission require simultaneous spectralimaging and polarimetric measurements, a solution could be to use two different telescopes for the two instruments. After all, even used with a telescope with a collecting area 10 times smaller than that of the stacked imager, the dedicated polarimeter would still require an observation time $\sim 90$ times shorter to reach the same MDP.

As a final note, it is worth mentioning that our results are not in agreement with those reported by other authors. In particular, Gouiffès et al. (2008) reported 

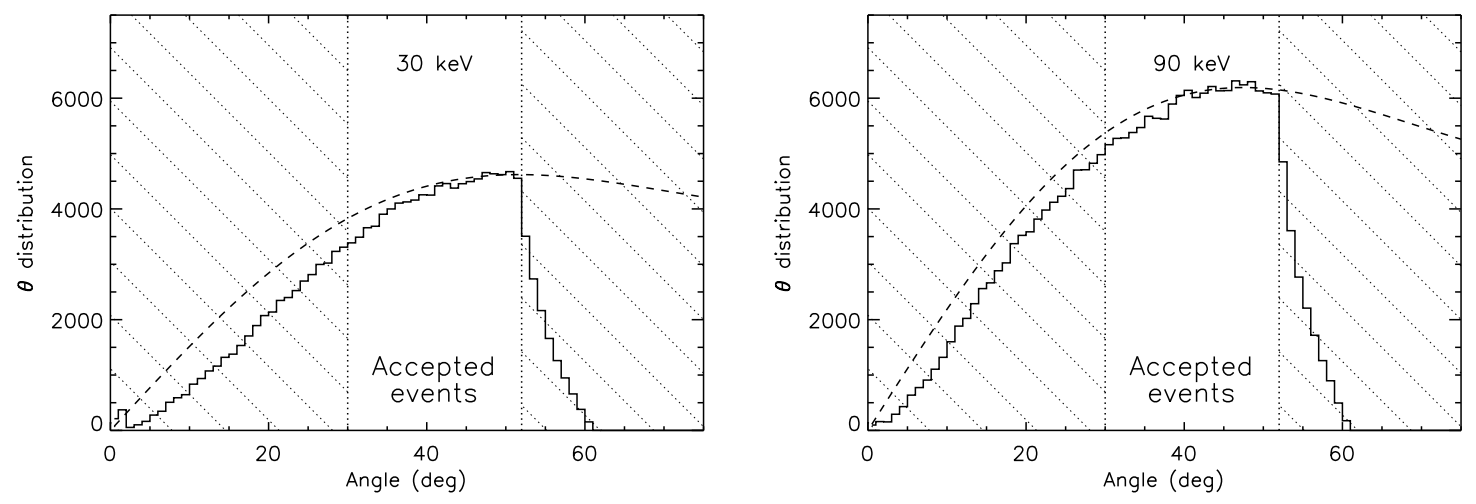

Figure 10. Comparison of the scattering angle distribution for the events which passed all the filters in Monte Carlo simulations at two energies, $30 \mathrm{keV}$ (left) and $90 \mathrm{keV}$ (right). The distributions are compared with that expected on the basis of the Klein-Nishina formula used in the analytical treatment (dashed line). The difference between the two distributions originate the small disagreement visible in Figure 9. The diagonally dashed areas are the regions which are cut out by the $\theta$-filter.

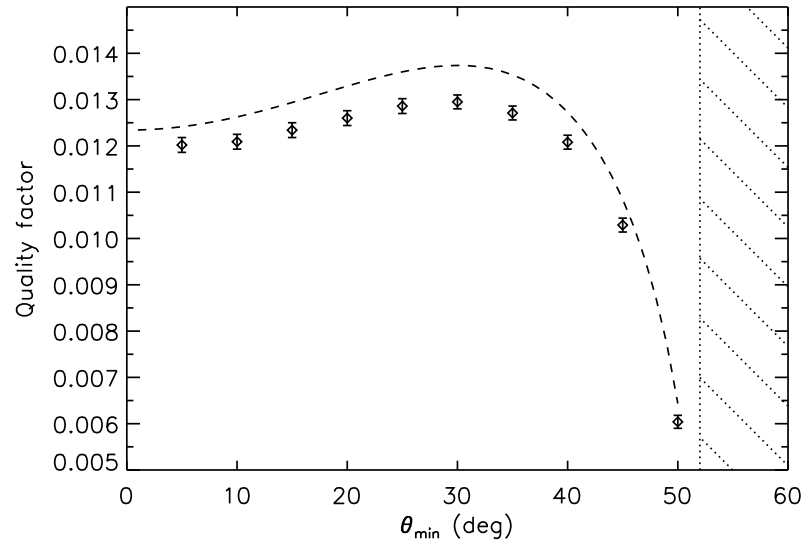

Figure 11. Quality factor as a function of $\theta_{\min }$ for $\theta_{\max }=52^{\circ}$ and $30 \mathrm{keV}$ photons. The dependence derived by the Monte Carlo (points) is in very good agreement with that expected from our analytical treatment and confirm a maximum sensitivity for $\theta_{\min }=$ $30^{\circ}$. The small offset can be ascribed as above to the fact that in Monte Carlo simulations we included the scattering function.

for the stacked imagers on-board Simbol-X a 35\% MDP for $100 \mathrm{mCrab}$ sources in $10 \mathrm{ks}$ between 20 and $80 \mathrm{keV}$, which corresponds to $11 \% \mathrm{MDP}$ for $100 \mathrm{mCrab}$ sources in $100 \mathrm{ks}$. Using the values derived by the Monte Carlo simulations (see Fig. 9) and without adding any background, we derived a MDP $\sim 28 \%$ in the latter case, that is a factor $\sim(28 / 11)^{2} \approx 6$ worse in observation time. For the COSPIX mission, Ferrando et al. (2010) reported a $0.7 \% \mathrm{MDP}$ for $100 \mathrm{mCrab}$ sources in $100 \mathrm{ks}$ between $20 \mathrm{keV}$ and $40 \mathrm{keV}$, while our estimate is $18 \%$. Incidentally, a dedicated polarimeter such as that presented by Soffitta et al. (2010) in the focus of the COSPIX telescope would reach a MDP $0.3 \%$ for $100 \mathrm{mCrab}$ sources in $100 \mathrm{ks}$. It is difficult to establish the origin of the disagreement between our results and those presented by previous authors because the value of the modulation factor, of the efficiency and of the background assumed to derive the MDP were not declared. Nevertheless, these disagreements can not be imputed only to the different geometry of the assumed detector, since our analysis suggests a rather weak dependency on parameters such as the distance between the detection plane or the size of the detectors.
FM and RC acknowledges the financial support on the INAF contract PRIN-INAF-2009. The authors are grateful to the anonymous referee for his/her interesting comments to this work.

\section{REFERENCES}

Agostinelli, S., Allison, J., Amako, K., et al. 2003, Nuclear Instruments and Methods in Physics Research Section A, 506, 250

Barret, D., Bavdaz, M., Budtz-Jorgensen, C., et al. 2003, The XEUS instruments (ESA Publications Division)

Bellazzini, R., Costa, E., Matt, G., \& Tagliaferri, G. 2010, X-ray Polarimetry: A New Window in Astrophysics (Cambridge University Press)

Bellazzini, R., \& Spandre, G. 2010, in X-ray Polarimetry: A New Window in Astrophysics (Cambridge University Press)

Bergstrom, P., \& Pratt, R. H. 1997, Radiation Physics and Chemistry, 50, 3

Black, J. K., Baker, R. G., Deines-Jones, P., Hill, J. E., \& Jahoda, K. 2007, Nuclear Instruments and Methods in Physics Research A, 581, 755

Black, J. K., Deines-Jones, P., Hill, J. E., et al. 2010, in Society of Photo-Optical Instrumentation Engineers (SPIE) Conference Series, Vol. 7732, Proc. of SPIE, 77320X

Catalano, O., Argan, A., Bellazzini, R., et al. 2010, in Proc. of SPIE, Vol. 7732, 773219

Christensen, F. E., Hornstrup, A., Westergaard, N. J., et al. 1992, in Society of Photo-Optical Instrumentation Engineers (SPIE) Conference Series, Vol. 1546, Proc. of SPIE, ed. R. B. Hoover, 160

Coburn, W., \& Boggs, S. E. 2003, Nature, 423, 415

Costa, E., Soffitta, P., Bellazzini, R., et al. 2001, Nature, 411, 662

Dean, A. J., Clark, D. J., Stephen, J. B., et al. 2008, Science, 321, 1183

Ferrando, P., Goldwurm, A., Laurent, P., et al. 2010, in 25th

Texas Symposium on Relativistic Astrophysics

Forot, M., Laurent, P., Grenier, I. A., Gouiffès, C., \& Lebrun, F. 2008, ApJ, 688, L29

Gouiffès, C., Laurent, P., \& Ferrando, P. 2008, in Polarimetry days in Rome: Crab status, theory and prospects

Heitler, W. 1954, Quantum theory of radiation (International Series of Monographs on Physics, Oxford: Clarendon, 1954, 3rd ed.)

Hubbell, J. 1997, Radiation Physics and Chemistry, 50, 113

Hubbell, J., Veigele, W., \& Briggs, E. 1975, Journal of physical and chemical reference data, 4, 471

Jahoda, K. 2010, in Society of Photo-Optical Instrumentation Engineers (SPIE) Conference Series, Vol. 7732, Proc. of SPIE

Kalemci, E., Boggs, S. E., Kouveliotou, C., Finger, M., \& Baring, M. G. 2007, ApJS, 169, 75

Klein, O., \& Nishina, T. 1929, Zeitschrift fur Physik, 52, 853

Krawczynski, H., Garson, A., Guo, Q., et al. 2011, Astroparticle Physics, 34, 550 
Lechner, P., Amoros, C., Barret, D., et al. 2010, in Proc. of SPIE, Vol. 7742, 77420W

Matt, G., Feroci, M., Rapisarda, M., \& Costa, E. 1996, Radiation Physics and Chemistry, 48, 403

McConnell, M. L. 2010, in X-ray Polarimetry: A New Window in Astrophysics (Cambridge University Press)

Muleri, F., Bellazzini, R., Costa, E., et al. 2006, in Proc. of SPIE, Vol. $6266,62662 \mathrm{X}$

Novick, R. 1975, Space Science Reviews, 18, 389

Novick, R., Weisskopf, M. C., Berthelsdorf, R., Linke, R., \& Wolff, R. S. 1972, ApJ, 174, L1

Pareschi, G., \& Cotroneo, V. 2003, in Proc. of SPIE, Vol. 5168, 53

Rutledge, R. E., \& Fox, D. B. 2004, MNRAS, 350, 1288
Soffitta, P., Costa, E., Muleri, F., et al. 2010, in Presented at the Society of Photo-Optical Instrumentation Engineers (SPIE) Conference, Vol. 7732, Proc. of SPIE, 77321A

Stefanescu, A., Bautz, M. W., Burrows, D. N., et al. 2010, NIMA, 624,533

Veigele, W., \& Tracy, P. 1966, American Journal of Physics Weisskopf, M. C., Cohen, G. G., Kestenbaum, H. L., et al. 1976, ApJ, 208, L125

Weisskopf, M. C., Elsner, R. F., \& O'Dell, S. L. 2010, in Proc. of SPIE, Vol. 7732, 77320E

Weisskopf, M. C., Silver, E. H., Kestenbaum, H. L., Long, K. S., \& Novick, R. 1978, ApJ, 220, L117

Willis, D. R., Barlow, E. J., Bird, A. J., et al. 2005, A\&A, 439, 245

Yonetoku, D., Murakami, T., Gunji, S., et al. 2011a, ApJ, 743, L30

—. 2011b, PASJ, 63, 625 